\title{
ALGEBRAIC INVARIANTS OF BOUNDARY LINKS
}

\author{
BY
}

NOBUYUKI SATO

\begin{abstract}
In this paper we study the homology of the universal abelian cover of the complement of a boundary link of $n$-spheres in $S^{n+2}$, as modules over the (free abelian) group of covering transformations. A consequence of our results is a characterization of the polynomial invariants $p_{i, q}$ of boundary links for $1<q<$ $[n / 2]$. Along the way we address the following algebraic problem: given a homomorphism of commutative rings $f: R \rightarrow S$ and a chain complex $C_{*}$ over $R$, determine when the complex $S \otimes_{R} C_{*}$ is acyclic. The present work is a step toward the characterization of link modules in general.
\end{abstract}

Introduction. A link $L$ of $n$-spheres in $S^{n+2}$ is an embedding $L: \amalg_{i=1}^{m} S_{i}^{n} \rightarrow S^{n+2}$, where $\amalg_{i=1}^{m} S_{i}^{n}$ represents the disjoint union of $m$ copies of $S^{n}$. The components of the link are the submanifolds $L\left(S_{i}^{n}\right)$. If the embedding $L$ extends to an embedding $\bar{L}: \amalg_{i=1}^{m} V_{i} \rightarrow S^{n+2}$ where $V_{i}$ is a manifold with boundary $S_{i}^{n}$, the link is called a boundary link. It is well known that the tubular neighborhoods of the components are trivial, and thus diffeomorphic to $S^{n} \times D^{2}$. Let $\mu_{i}$ be the circle represented by $* \times \partial D^{2}$ in $S_{i}^{n} \times D^{2}$, oriented so that $\mu_{i}$ links $S_{i}^{n}$ with linking number +1 . The $\mu_{i}$ are called the meridians.

Many of the classical link invariants come from looking at certain algebraic invariants of the complement $X=S^{n+2}-\cup_{i=1}^{m} N\left(L\left(S_{i}^{n}\right)\right)$, where $N\left(L\left(S_{i}^{n}\right)\right)$ is an open tubular neighborhood of $L\left(S_{i}^{n}\right)$ in $S^{n+2}$. These include the Alexander polynomials, the higher polynomial invariants, and the Alexander ideals. These latter are all related to the homology of the universal abelian cover $\tilde{X}$ of $X$. In 1977, Levine [14] characterized the homology of $\tilde{X}$ as a module over the covering transformations (except for $H_{1} \tilde{X}$ ) for knots, generalizing earlier works on the characterization of knot polynomials ([19], [11] and [12]).

The present work is a step toward the characterization of $H_{*} \tilde{X}$ for links, with applications to the link polynomials. We work with the case of boundary links, where it will be seen that the algebra is somewhat easier than the general case.

Our main results are the finding of certain necessary conditions on $H_{*} \tilde{X}$ (Theorems 2.7 and 3.1 and Corollaries 4.6 and 4.8). This is followed by the finding of sufficient conditions and the construction of boundary links with certain specified $H_{*} \tilde{X}$ (Theorem 5.1). In $\S 6$, this is re-interpreted in terms of the polynomial invariants, to obtain a characterization of these invariants below the middle dimension for a boundary link of $n$-spheres, $n>2$.

Received by the editors February 6, 1979.

AMS (MOS) subject classifications (1970). Primary 57C45; Secondary 13D99, 18G10.

Key words and phrases. Link, boundary link, universal abelian cover, module of type $L$. 
Since, by Alexander duality, the group $H_{1} X$ is free abelian of rank $m$, the group of covering transformations of $\tilde{X}$ is $\mathbf{Z}^{m}$. Hence, the groups $H_{*} \tilde{X}$ are modules over $\Lambda=\mathbf{Z}\left[\mathbf{Z}^{m}\right]$, which we identify with the localized polynomial ring $\mathbf{Z}\left[x_{1}, \ldots, x_{m}, x_{1}^{-1}, \ldots, x_{m}^{-1}\right]$ by writing $\mathbf{Z}^{m}$ multiplicatively. This ring comes equipped with a canonical augmentation $\varepsilon: \Lambda \rightarrow \mathbf{Z}$ defined by sending $\Sigma_{g \in \mathbf{Z}^{m}} n_{g} g$ to $\sum n_{g}$.

The paper is organized as follows. $\$ 1$ deals with the central algebraic problem of the paper, in some generality, and may be of independent interest. $\$ \S 2$ through 4 develop necessary conditions on $H_{*} \tilde{X}$, while sufficient conditions are found in $\S 5$. As noted before, these theorems are interpreted as theorems concerning the polynomial invariants in $\S 6$. In $\$ 4$ we show that simple boundary links satisfy a high-dimensional analogue of Blanchfield duality.

Some of the ideas in this work come from my Ph.D. thesis, written at Brandeis University. I am grateful for the guidance of Jerome Levine.

1. Modules which are $f$-trivial and $f$-acyclicity. In this section we develop the main algebraic concept of this paper, in some generality. Let $f: R \rightarrow S$ be a map of commutative rings with unit, and let $C_{*}$ be a chain complex over $R$. We ask what conditions to place on the homology of $C_{*}$ so that $S \otimes_{R} C_{*}$ is acyclic, that is, so that $C_{*}$ is $f$-acyclic. For links of $n$-spheres in $S^{n+2}$, the chain complex of the universal abelian cover $\tilde{X}$ of the complement $X$ is a chain complex over $\Lambda$, and the augmentation map gives a map of rings $\varepsilon: \Lambda \rightarrow Z$. The chains of $X$ are given by $\mathbf{Z} \otimes_{\Lambda} C_{*}(\tilde{X})$, and $H_{q} X \cong 0$ for $1<q<n+1$. We wish to see what conditions this imposes on $H_{*} \tilde{X}$. We will wish to place some restrictions on our general situation. We will assume that for each $q, C_{q}$ is a flat $R$-module, that $C_{q} \simeq 0$ for $q<0$, and that the homology of $C_{*}$ is finitely generated in each dimension. This will be automatic for the case of links.

A finitely generated $R$-module $M$ is called $f$-trivial if $\operatorname{Tor}_{q}^{R}(S, M) \simeq 0$ for all $q$. In the special case of links where $f: R \rightarrow S$ is given by $\varepsilon: \Lambda \rightarrow \mathbf{Z}$, we say that an $\varepsilon$-trivial $\Lambda$-module is a module of type $L$. It turns out that, in many cases, the condition that $\operatorname{Tor}_{q}^{R}(S, M) \cong 0$ for all $q$ may be replaced by the seemingly weaker condition that $\operatorname{Tor}_{0}^{R}(S, M) \cong 0$, that is, that $S \otimes_{R} M \cong 0$. The following result is related to a result of Dwyer [5]. Justin Smith has obtained similar results in a more general setting [23]. It is true in more generality than it is stated; I do not know whether it is true in complete generality. Here, $M$ is a finitely generated $R$-module.

THEOREM 1.1. If either of the following conditions is true, then $M$ is $f$-trivial if and only if $S \otimes_{R} M \cong 0$.

(i) $f: R \rightarrow S$ is onto.

(ii) $R$ and $S$ are noetherian rings and $M$ has a finite free resolution over $R$. This condition means that there is an exact sequence $0 \rightarrow F_{n} \rightarrow F_{n-1} \rightarrow \cdots \rightarrow F_{1} \rightarrow F_{0}$ $\rightarrow M \rightarrow 0$ where $F_{i}$ is a free $R$-module of finite rank for each $i$.

Remark. For the case of $\varepsilon: \Lambda \rightarrow \mathbf{Z}$, both conditions are satisfied. Clearly, (i) is satisfied. To see (ii), we note that $\Lambda$ has finite global dimension and every projective $\Lambda$-module is free [22]. 
Proof of 1.1. (i) We use an argument similar to one given in [5]. Let $I=\operatorname{ker} f$. Then the condition that $S \otimes_{R} M \cong 0$ is equivalent to the statement that $M=I M$. By Nakayama's lemma [15 p. 11] there exists an element $r \in R$ of the form $1+i$, where $i \in I$, such that $r M=0$. We will show that multiplication by $r$ is both the identity map and the zero map on $\operatorname{Tor}_{q}^{R}(S, M), q>0$. This will prove that these modules are trivial.

Multiplication by $r$ is induced by multiplication by $r$ on $S$; since $f(r)=1$, this induces the identity map. On the other hand, it is also induced by multiplication by $r$ on $M$, which is the zero map. Hence, on $\operatorname{Tor}_{q}^{R}(S, M)$, multiplication by $r$ is both the identity and zero, making $\operatorname{Tor}_{q}^{R}(S, M) \cong 0$.

For 1.1 part (ii) we need the Buchsbaum-Eisenbud theory of finite free resolutions (see [2] and [3]; see also [17] for a different approach). For the convenience of the reader and because we will use some of this material later, we will summarize the portions of the theory we will need. We now assume that $R$ and $S$ are noetherian rings.

Let $g: F \rightarrow G$ be a map where $F$ is a free $R$-module of rank $m$ and $G$ is a free $R$-module of rank $n$. Choosing bases for $F$ and $G$, we may represent $g$ by an $(m \times n)$ matrix over $R$. For $i \geqslant 0$, let $I_{i}(g)$ be the ideal generated by the $(n-i) \times$ $(n-i)$ minor determinants of this matrix. If $n>m$, then $I_{i}=0$ for $0<i<n-m$ and if $i$ is larger than $m$ or $n$, take $I_{i}=(1)$. By the process of expansion by minors, $I_{k} \subseteq I_{k+1}$ for all $k$. It is not difficult to see that these ideals are independent of the choice of bases for $F$ and $G$; in fact, they are the Fitting invariants of cok $g$ (see [9, p. 145]).

The rank of $g$, denoted $\operatorname{rk}(g)$, is the largest $k$ for which there exists a nonzero $k \times k$ minor determinant. The ideal $I(g)$ of $k \times k$ minor determinants, where $k=\mathrm{rk}(g)$, is the first nonvanishing Fitting invariant of cok $g$.

The depth of an ideal $A$ is the length of the longest $R$-sequence in $A$. This makes sense because $R$ is noetherian. If $A=R$, then $\operatorname{depth}(A)=\infty$. The radical of an ideal $A$, denoted $\operatorname{Rad}(A)$, is the set of all $r \in R$ such that some power of $r$ is in $A$.

THeOREM 1.2. Suppose we are given a complex $\mathbf{F}$ where $F_{k}$ is a free module of finite rank for each $k$ :

$$
\mathbf{F}: 0 \rightarrow F_{n} \underset{\varphi_{n}}{\rightarrow} F_{n-1} \underset{\varphi_{n-1}}{\rightarrow} \cdots \rightarrow F_{1} \underset{\varphi_{1}}{\rightarrow} F_{0} \text {. }
$$

(i) If $\mathbf{F}$ is exact, then for each $k, \operatorname{Rad}\left(I\left(\varphi_{k}\right)\right) \subseteq \operatorname{Rad}\left(I\left(\varphi_{k+1}\right)\right)$.

(ii) $\mathbf{F}$ is exact if and only if for each $k, \operatorname{rk}\left(\varphi_{k}\right)+\operatorname{rk}\left(\varphi_{k+1}\right)=\operatorname{rk}\left(F_{k}\right)$ and $\operatorname{depth}\left(I\left(\varphi_{k}\right)\right) \geqslant k$.

Part (i) is [3, Theorem 2.1] and part (ii) is [2, Corollary 1].

Proof of 1.1. (ii) Suppose that $M$ has a finite free resolution $\mathbf{F}$, and that $S \otimes_{R} M \cong 0$. It follows from [2, Lemma 1] that the ideal generated by $f\left(I\left(\varphi_{1}\right)\right)$ is $S$. We wish to show that $S \otimes_{R} \mathbf{F}$ is exact. This will be accomplished, for example, if we can show that $\operatorname{rk}\left(1 \otimes \varphi_{k}\right)=\operatorname{rk}\left(\varphi_{k}\right)$ and $I\left(1 \otimes \varphi_{k}\right)=S$ for all $k$. Then we apply Theorem 1.2 part (ii). Since the rank of $1 \otimes \varphi_{k}$ can never exceed the rank of 
$\varphi_{k}$ (a matrix representative for $1 \otimes \varphi_{k}$ is obtained by mapping a matrix representative for $\varphi_{k}$ via $f$ to $S$ ), both statements will follow from 1.2(i) and the following lemma.

LemmA 1.3. Suppose $I$ and $J$ are ideals of $R$, with $\operatorname{Rad}(I) \subseteq \operatorname{Rad}(J)$. If the ideal of $S$ generated by $f(I)$ is $S$, then so is the ideal generated by $f(J)$.

Proof. For any subset $K$ of $S$, let $\{K\}$ denote the ideal of $S$ generated by $K$. Clearly, if the radical of an ideal is the whole ring, then the ideal itself must be the whole ring. Therefore, it suffices to prove that $\operatorname{Rad}(\{f(J)\})=S$. On the other hand, $f(\operatorname{Rad}(J)) \subseteq \operatorname{Rad}\{f(J)\}$. Thus, it suffices to show that $\{f(\operatorname{Rad}(J))\}=S$. But $I \subseteq \operatorname{Rad}(I) \subseteq \operatorname{Rad}(J)$, so that $\{f(I)\} \subseteq\{f(\operatorname{Rad}(J))\}$. Since $\{f(I)\}=S$, we are done.

This completes the proof of Theorem 1.1. We now turn to the main result of this section. It is, as is 1.1 , true in greater generality.

THEOREM 1.4. $C_{*}$ is $f$-acyclic if and only if for each $q, H_{q}\left(C_{*}\right)$ is f-trivial, as long as one of the conditions below holds.

(i) $f: R \rightarrow S$ is onto.

(ii) $R$ and $S$ are neotherian rings and for each $q, H_{q}\left(C_{*}\right)$ has a finite free resolution over $R$.

The proof depends on the following universal coefficient spectral sequence for homology. A special case of it is the Cartan-Leray-Serre spectral sequence of a covering projection. See, for example, [8].

THEOREM 1.5. Let $C_{*}$ be a chain complex of flat $R$-modules, and let $M$ be any $R$-module. There is a spectral sequence $\left(E^{r}, d^{r}\right)$ converging to the homology $H_{*}\left(M \otimes_{R} C_{*}\right)$, with $E_{p, q}^{2} \cong \operatorname{Tor}_{P}\left(M, H_{q}\left(C_{*}\right)\right)$ and differential $d^{r}$ of degree $(-r$, $r-1)$.

Proof of Theorem 1.4. Suppose that either (i) or (ii) holds. If $H_{q}\left(C_{*}\right)$ is $f$-trivial, we apply the spectral sequence with $M=S . E_{p, q}^{2} \cong 0$ for all $p, q$ so that $H_{*}\left(S \otimes_{R} C_{*}\right) \cong 0$ in all dimensions. Hence $C_{*}$ is $f$-acyclic. If, conversely, $C_{*}$ is $f$-acyclic, we know that $E_{p, q}^{\infty} \cong 0$ for all $p, q$. Since $E_{0,0}^{2} \simeq E_{0,0}^{\infty}$, we see that $S \otimes_{R} H_{0}\left(C_{*}\right) \cong 0$. Hence, by Theorem $1.1, E_{p, 0}^{2} \cong 0$ for all $p$. Then $E_{0,1}^{2} \simeq E_{0,1}^{\infty}$. Repeating the argument, we see that $H_{1}\left(C_{*}\right)$ is $f$-trivial. But we can keep applying this argument to show that $H_{q}\left(C_{*}\right)$ is $f$-trivial for every $q$.

REMARK. A slight variation of this argument will be used in $\$ 3$ to prove Theorem 3.1.

We finish with a "matrix-theoretic" characterization of $\Lambda$-modules of type $L$, and construct examples.

THeOREM 1.6. A finitely generated $\Lambda$-module $M$ is of type $L$ if and only if $\varepsilon\left(I_{0}(M)\right)=(1)=\mathbf{Z}$, where $\varepsilon: \Lambda \rightarrow \mathbf{Z}$ is the augmentation. In other words, $M$ is of type $L$ if and only if it has a presentation matrix $A$ such that the minors of largest size of the integral matrix $\varepsilon(A)$ are relatively prime. 
EXAmple. Let $A=\left(a_{i j}\right)$ be a square matrix over $\Lambda$ such that $\varepsilon(\operatorname{det} A)= \pm 1$. The module presented by $A$ is of type $L$. In fact, all modules of type $L$ of projective dimension one arise in this fashion. Typical examples would be the module presented by the $1 \times 1$ matrix $(g)$ where $\varepsilon(g)= \pm 1$ (say $g=2 x_{1}-1$ ), or the module presented by a diagonal matrix whose diagonal entries satisfied the condition on $g$ above.

2. Necessary conditions on $H_{1} \tilde{X}$. In this section, we derive necessary conditions on a finitely generated $\Lambda$-module $M$ to be $H_{1} \tilde{X}$. We will see that $H_{1} \tilde{X}$ always has a special form.

Let $V=\bigvee_{i=1}^{m} S^{1}$, with vertex $v_{0}$. Choose a base point $x_{0}$ in the interior of the link complement $X$. We have the following result of Smythe [21] and Gutierrez [6]. Let $X$ be the complement of a boundary link.

Lemma 2.1. There are base-point preserving maps $e: V \rightarrow X$ and $r: X \rightarrow V$ such that e carries the loops of $V$ to the meridians of $X$ and $r$ carries the meridians of $X$ to the loops of $V$. The composition $r \circ e: V \rightarrow V$ is homotopic, rel $v_{0}$, to $\mathrm{id}_{V}$. In fact, the existence of such maps implies that the link is a boundary link.

An immediate corollary, also due to the aforementioned authors, is:

Corollary 2.2. If $\pi=\pi_{1}\left(X, x_{0}\right)$, then there is a splitting $F_{m} \rightarrow \pi \rightarrow F_{m}$ where $F_{m}$ is the free group on $m$ letters.

We can define a functor $\mathscr{F}$ from groups to Abelian groups by letting $\mathscr{F}(G)$ be $G^{\prime} / G^{\prime \prime}$ (the commutator subgroup modulo its commutator) and if $h: G \rightarrow H$ is any homomorphism, $\mathscr{F}(h)$ is the induced map $G^{\prime} / G^{\prime \prime} \rightarrow H^{\prime} / H^{\prime \prime}$. We have the immediate result:

COROLlary 2.3. There is an induced splitting $F_{m}^{\prime} / F_{m}^{\prime \prime} \rightarrow \pi^{\prime} / \pi^{\prime \prime} \rightarrow F_{m}^{\prime} / F_{m}^{\prime \prime}$.

It can easily be checked that these maps respect the action via conjugation by elements of $\pi / \pi^{\prime} \cong F_{m} / F_{m}^{\prime} \cong \mathbf{Z}^{m}$. Therefore, $\pi^{\prime} / \pi^{\prime \prime}$ splits as a $\Lambda$-module. We now have the following result:

Corollary 2.4. $H_{1} \tilde{X} \cong F_{m}^{\prime} / F_{m}^{\prime \prime} \oplus T$ for some $\Lambda$-module $T$.

We will see that $T$ is the torsion submodule of $H_{1} \tilde{X}$, and that $T$ is in fact of type $L$. First, however, we will get a description of $F_{m}^{\prime} / F_{m}^{\prime \prime}$.

LEMMA 2.5. $F_{m}^{\prime} / F_{m}^{\prime \prime}$ is the kernel of the map which sends the basis elements $e_{i}$ of $\Lambda^{m}$ to $x_{i}-1 \in \Lambda$. Thus, $F_{m}^{\prime} / F_{m}^{\prime \prime}$ is torsion-free of rank $m-1$ and has projective dimension $m-2$. In particular, it is free if and only if $m<2$.

Proof. The first statement follows from examining the chains of the universal abelian cover of $V$. The rest follows from the fact that the map sending the basis elements $e_{i}$ of $\Lambda^{m}$ to $x_{i}-1 \in \Lambda$ is the first map in the Koszul complex of the $\Lambda$-regular sequence $\left(x_{1}-1, x_{2}-1, \ldots, x_{m}-1\right)$, which is exact and of minimal length (see [15]). The complex is of length $m$. Therefore, $F_{m}^{\prime} / F_{m}^{\prime \prime}$ has a minimal 
projective resolution of length $m-2$ (note we are using the fact that all projective $\Lambda$-modules are free [22]). The rank follows from a Euler characteristic argument using the exact sequence $0 \rightarrow F_{m}^{\prime} / F_{m}^{\prime \prime} \rightarrow \Lambda^{m} \rightarrow \Lambda \rightarrow \Lambda /\left(x_{1}-1, \ldots, x_{m}-1\right) \rightarrow 0$.

We now prove the following, which gives a description of $T$.

LEMMA 2.6. $T$ is of type L. Hence $T$ is the torsion submodule of $H_{1} \tilde{X}$.

Proof. We use the Cartan-Leray-Serre spectral sequence of the covering $\tilde{X} \rightarrow X$. $E_{p, q}^{2} \cong \operatorname{Tor}_{p}^{\Lambda}\left(\mathrm{Z}, H_{q} \tilde{X}\right)$, which is isomorphic to $H_{p}\left(\mathbf{Z}^{m} ; H_{q} \tilde{X}\right)$. Let $\left(\begin{array}{c}a \\ b\end{array}\right)$ represent the binomial coefficient $(a !) / b !(a-b)$ !. For small $p, q$ we picture the $E^{2}$-term:

$$
\begin{aligned}
& q=1 \quad \mathrm{Z} \otimes_{\Lambda}\left(F_{m}^{\prime} / F_{m}^{\prime \prime} \oplus T\right) \\
& q=0 \quad \mathbf{Z}^{\left(\begin{array}{c}
m \\
0
\end{array}\right)} \quad \mathbf{Z}^{\left(\begin{array}{c}
m \\
1
\end{array}\right)} \quad \mathbf{Z}^{\left(\begin{array}{c}
m \\
2
\end{array}\right)} \\
& p=0 \quad p=1 \quad p=2
\end{aligned}
$$

Since $H_{1} X \cong \mathbf{Z}^{m}$, we see that $d_{2,0}^{2}$ must be onto. But $\mathbf{Z} \otimes_{\Lambda}\left(F_{m}^{\prime} / F_{m}^{\prime \prime} \oplus T\right) \cong(\mathbf{Z}$ $\left.\otimes_{\Lambda}\left(F_{m}^{\prime} / F_{m}^{\prime \prime}\right)\right) \oplus\left(\mathbf{Z} \otimes_{\Lambda} T\right)$ and it is easy to see that $\mathbf{Z} \otimes_{\Lambda}\left(F_{m}^{\prime} / F_{m}^{\prime \prime}\right) \simeq \mathbf{Z}^{(m)}$ (for example, all the differentials in the Koszul complex of the $\Lambda$-sequence $\left(x_{1}-\right.$ $\left.1, \ldots, x_{m}-1\right)$ become zero upon tensoring with $\mathbf{Z}$, so that $\mathbf{Z} \otimes_{\Lambda}\left(F_{m}^{\prime} / F_{m}^{\prime \prime}\right) \cong \mathbf{Z}$ $\left.\otimes_{\Lambda} \Lambda^{\left(\frac{m}{2}\right)} \cong \mathbf{Z}^{\left(\frac{m}{2}\right)}\right)$. We have $d_{2,0}^{2}: \mathbf{Z}^{\left(\frac{m}{2}\right)} \rightarrow \mathbf{Z}^{\left(\frac{m}{2}\right)} \oplus\left(\mathbf{Z} \otimes_{\Lambda} T\right)$ onto so that $\mathbf{Z} \otimes_{\Lambda} T \cong 0$. By Theorem 1.1, the lemma follows.

To summarize,

THEOREM 2.7. Suppose $\tilde{X}$ is the universal abelian cover of the complement of $a$ boundary link. Then

$$
H_{1} \tilde{X} \cong F_{m}^{\prime} / F_{m}^{\prime \prime} \oplus T
$$

where $T$ is the $\Lambda$-torsion submodule of $H_{1} \tilde{X}$ and is of type $L$.

It turns out that it will be useful to have the splitting of Theorem 2.7 be geometrically induced. First, we prove a result about covering spaces.

Suppose that $f:\left(X, x_{0}\right) \rightarrow\left(Y, y_{0}\right)$ is a map of $\mathrm{CW}$-complexes with base point. Let $G=\pi_{1}\left(X, x_{0}\right)$ and $H=\pi_{1}\left(Y, y_{0}\right)$, and let $K \triangleleft G, L \triangleleft H$ such that $f_{\sharp}(K) \subseteq L$. Let $\tilde{X}$ be the covering of $X$ associated to $K$ and $\tilde{Y}$ the covering of $Y$ associated to $L$. Let $f_{*}: G / K \rightarrow H / L$ be the induced map from $f_{\sharp}: G \rightarrow H$. Note that $G / K$ is the group of covering transformations of $\tilde{X}$, and $H / L$ is the group of covering transformations of $\tilde{Y}$. Choose base points $\tilde{x}_{0} \in p^{-1}\left(x_{0}\right)$ and $\tilde{y}_{0} \in p^{-1}\left(y_{0}\right)$. Then there is a unique lift of $f$ to $\tilde{f}:\left(\tilde{X}, \tilde{x}_{0}\right) \rightarrow\left(\tilde{Y}, \tilde{y}_{0}\right)$.

Proposition 2.8. For any covering transformation $g \tilde{f}\left(g \cdot \tilde{x}_{0}\right)=f_{*}(g) \cdot \tilde{y}_{0}$. Thus, the unique lift is "equivariant" with respect to the covering transformations.

Proof. Let $\gamma$ be a path from $\tilde{x}_{0}$ to $g \cdot \tilde{x}_{0}$ in $\tilde{X}$. Then $\tilde{f} \circ \gamma$ is a path from $\tilde{y}_{0}$ to $g^{\prime} \cdot \tilde{y}_{0}$ for some covering transformation $g^{\prime}$. Projecting $\gamma$ to $x$ gives a loop at $x_{0} \in X$ representing a class $[\gamma]$ which represents $g$ in $G / K$. But projecting $\tilde{f} \circ \gamma$ to $Y$ gives a loop which represents $f_{*} g$ in $H / L$. Hence, $g^{\prime}=f_{*} g$ and the result is proved. 
Now, let $\tilde{V}$ and $\tilde{X}$ be the universal abelian covers of $V$ and $X$, respectively. Choose base points $\tilde{x}_{0} \in \tilde{X}$ and $\tilde{v}_{0} \in \tilde{V}$. Let $\tilde{e}$ be the unique lift of $e$ and let $\tilde{r}$ be the unique lift of $r$. Since $e$ and $r$ induce canonical identifications of $H_{1} X$, which is the group of covering transformations, $\tilde{e}$ and $\tilde{r}$ are equivariant maps. Since $r \circ e \cong \mathrm{id}_{V}$ rel $v_{0}$, we have that $\tilde{r} \circ \tilde{e}$ is homotopic to a covering transformation rel $\tilde{v}_{0}$. Hence, $\tilde{r} \circ \tilde{e} \cong \mathrm{id}_{\tilde{V}}$. This induces splittings on all homology. In particular, we have $\tilde{e}_{*}$ is a monomorphism and so $H_{1} \tilde{X} \cong \tilde{e}_{*} H_{1} \tilde{V} \oplus T$ for some module $T$. But $H_{1} \tilde{V} \cong F_{m}^{\prime} / F_{m}^{\prime \prime}$.

3. A necessary condition on the higher homology of $\tilde{X}$. The results of the previous section are valid for all $n$. We now restrict our attention to higher dimensional boundary links, where $n>1$. The main result of this section is the following:

THEOREM 3.1. $H_{q} \tilde{X}$ is of type $L$ for $2 \leqslant q \leqslant n$.

The proof will proceed by steps. We first figure out the $d^{2}$ differentials in the Cartan-Leray-Serre spectral sequence of the covering $\tilde{X} \rightarrow X$. The $E^{2}$-term is given by $E_{p, q}^{2} \cong H_{p}\left(\mathbf{Z}^{m} ; H_{q} \tilde{X}\right)$ with differential $d_{p, q}^{2}: E_{p, q}^{2} \rightarrow E_{p-2, q+1}^{2}$. For details, see for example [8, p. 464].

By Proposition 2.8, the maps $\tilde{e}: \tilde{V} \rightarrow \tilde{X}$ and $\tilde{r}: \tilde{X} \rightarrow \tilde{V}$ give rise to equivariant chain maps and hence maps of spectral sequences of the covers $\tilde{V} \rightarrow V$ and $\tilde{X} \rightarrow X$. We will use this information to determine the differentials. First, we calculate the groups $E_{p, q}^{2}$ for $q=0$ and $q=1$.

Proposition 3.2. (i) $E_{p, 0}^{2} \cong \mathbf{Z}^{(m)}$ for $0 \leqslant p \leqslant m$, and $E_{p, 0}^{2} \simeq 0$ for $p>m$.

(ii) $E_{p, 1}^{2} \cong \mathbf{Z}^{\left({ }_{p+2}^{m}\right)}$ for $0 \leqslant p \leqslant m-2$, and $E_{p, 1}^{2} \cong 0$ for $p>m-2$.

Proof. $E_{p, q}^{2} \cong \operatorname{Tor}_{p}^{\Lambda}\left(\mathbf{Z}, H_{q} \tilde{X}\right)$, so what we need is an explicit resolution of $H_{0} \tilde{X}$ and $H_{1} \tilde{X}$. However, since $H_{1} \tilde{X} \cong F_{m}^{\prime} / F_{m}^{\prime \prime} \oplus T$ where $T$ is of type $L$, we need only resolve $F_{m}^{\prime} / F_{m}^{\prime \prime}$. We resolve $H_{0} \tilde{X} \cong \mathbf{Z} \cong \Lambda /\left(x_{1}-1, \ldots, x_{m}-1\right)$ by the Koszul complex, which is exact:

$$
0 \rightarrow \Lambda^{\left(\begin{array}{c}
m \\
m
\end{array}\right)} \underset{d_{m}}{\rightarrow} \Lambda^{\left(\begin{array}{c}
m \\
m-1
\end{array}\right)} \rightarrow \cdots \rightarrow \Lambda^{\left(\begin{array}{c}
m \\
2
\end{array}\right)} \underset{d_{2}}{\rightarrow} \Lambda^{\left(\begin{array}{c}
m \\
1
\end{array}\right)} \underset{d_{1}}{\rightarrow} \Lambda \rightarrow \mathbf{Z} \rightarrow 0
$$

The maps $d_{k}$ have the property that $1 \otimes_{\Lambda} d_{k}: \mathbf{Z}^{\left(\begin{array}{c}m \\ k\end{array}\right)} \rightarrow \mathbf{Z}^{\left(\begin{array}{c}m \\ k-1\end{array}\right)}$ are all zero. This proves part (i). To see part (ii), note that by Lemma $2.5, F_{m}^{\prime} / F_{m}^{\prime \prime} \cong \operatorname{ker} d_{1}=\operatorname{im} d_{2}$. This completes the argument.

Thus, the maps $d_{p, 0}^{2}$ for $p \geqslant 2$ are maps of groups which are abstractly isomorphic. We need to see something more specific, namely, the content of the next proposition.

PROPOSITION 3.3. The maps $d_{p, 0}^{2}$ are isomorphisms for $p \geqslant 2$.

Proof. The map $\tilde{e}: \tilde{V} \rightarrow \tilde{X}$ induces a map of $E^{2}$-terms of the corresponding spectral sequence of coverings. By the discussion at the end of $\S 2, \tilde{e}_{*}$ induces isomorphisms of the bottom two rows. Thus, for $p \geqslant 2$, there are commuting 
diagrams

$$
\begin{array}{ccc}
\operatorname{Tor}_{p-2}^{\Lambda}\left(\mathbf{Z}, H_{1} \tilde{V}\right) & \stackrel{\tilde{e}_{*}}{\rightarrow} & \operatorname{Tor}_{p-2}^{\Lambda}\left(\mathbf{Z}, H_{1} \tilde{X}\right) \\
\uparrow d_{p, 0}^{2} & & \uparrow d_{p, 0}^{2} \\
\operatorname{Tor}_{p}^{\Lambda}\left(\mathbf{Z}, H_{0} \tilde{V}\right) & \underset{\tilde{e}_{*}}{\rightarrow} & \operatorname{Tor}_{p}^{\Lambda}\left(\mathbf{Z}, H_{0} \tilde{X}\right)
\end{array}
$$

where the two horizontal maps are isomorphisms. Since $H_{*} V \cong 0$ in dimensions greater than one, the left-hand maps are isomorphisms for trivial dimensional reasons. Hence, the right-hand vertical maps are isomorphisms. This completes the proof of 3.3 .

We can now prove the theorem. Proposition 3.3 implies that $E_{0,2}^{2} \simeq E_{0,2}^{\infty} \cong H_{2} X$. But $H_{2} X \cong 0$ and $E_{0,2}^{2} \cong \mathrm{Z} \otimes_{\Lambda} H_{2} \tilde{X}$. Hence, $H_{2} \tilde{X}$ is of type $L$, and so $E_{p, 2}^{2} \simeq 0$ for all $p$. As long as $H_{k} X \cong 0$, that is, for $k \leqslant n$, we may repeat the argument, showing that $\mathbf{Z} \otimes_{\Lambda} H_{k} \tilde{X} \cong 0$. This completes the proof of Theorem 3.1. We have also proved

Corollary 3.4. $\mathbf{Z} \otimes_{\Lambda} H_{n+1} \tilde{X} \cong \mathbf{Z}^{m-1}$ and $\operatorname{Tor}_{q}^{\Lambda}\left(\mathbf{Z}, H_{n+1} \tilde{X}\right) \cong 0$ for all $q>0$.

RemarK. Theorem 3.1 is actually true for links in general, and not just for boundary links. This follows from a different argument, which will appear in a future paper.

4. Simple odd-dimensional boundary links. A boundary link of dimension $n=2 q$ $-1, q \geqslant 2$, is called simple if $\pi_{k} X \cong \pi_{k} V$ for $k<q$ and $\pi_{1} X$ is freely generated by the meridians (recall $V=\bigvee_{i=1}^{m} S^{1}$ ). In this section, we will show that simplicity imposes some severe additional restrictions on $H_{q} \tilde{X}$. Specifically, we will show that, as a $\Lambda$-module, $H_{q} \tilde{X}$ has a presentation matrix which is square and whose determinant $\Delta$ satisfies $\varepsilon(\Delta)= \pm 1$, and that $H_{q} \tilde{X}$ supports a skew-Hermitian pairing into $\Lambda_{0} / \Lambda\left(\Lambda_{0}\right.$ is the quotient field of $\left.\Lambda\right)$ which is the higher dimensional analogue of the Blanchfield pairing [1].

It is useful, in the case of a simple boundary link, to consider a certain closed manifold $M$ which is closely related to $X$. Let $f_{1}, \ldots, f_{m}: S^{n} \rightarrow S^{n+2}$ be embeddings whose images are the components of the link. These extend to embeddings $\varphi_{1}, \ldots, \varphi_{m}: S^{n} \times D^{2} \rightarrow S^{n+2}$ by the triviality of the normal bundles. Surgery on these embeddings produces a closed $(n+2)$-dimensional manifold $M$ and embeddings $\psi_{1}, \ldots, \psi_{m}: D^{n+1} \times S^{1} \rightarrow M$ with disjoint images. Since $M=X \cup$ $\left(\cup_{i=1}^{m}\right.$ im $\left.\psi_{i}\right)$, the pair $(M, X)$ is $n$-connected. Thus, it suffices to study $H_{q} \tilde{M}$, where $\tilde{M}$ is the universal abelian cover of $M$. The following lemma, essentially due to Kervaire (see [10, Lemma II.9]), shows why we use $M$.

LEMMA 4.1. There is a handle decomposition of $M$ which consists of one handle of index $0, m$ handles of index $1, m$ handles of index $(n+1)$, one handle of index $(n+2)$, and no handles of index $k$ for $2 \leqslant k<q$ and $q+1<k \leqslant n$.

This is proved using standard "handle-trading" arguments. One might prove this, for example, by making the necessary adjustments to the proof of [10, Lemma II.9], which is the same statement except that $m=1$. 
To analyze $H_{*} \tilde{M}$ and $H_{*} \tilde{X}$, we will need to use the universal coefficient spectral sequence (see [14] for a construction of this spectral sequence). We will copy the statement of existence from $[14, \S 2]$, for the convenience of the reader.

Let $R, S$ be rings with $1, C_{*}$ a free left chain complex over $\mathbf{R}$ and $G$ an $(R-S)$-bimodule. Then $H^{*}\left(C_{*} ; G\right)=H_{*}\left(\operatorname{Hom}_{\mathbf{R}}\left(C_{*}, G\right)\right)$ is a graded right $S$ module, as are $\operatorname{Hom}_{R}\left(H_{*}\left(C_{*}\right), G\right)$ and, in general, $\operatorname{Ext}_{R}^{q}\left(H_{*}\left(C_{*}\right), G\right)$.

These are related by a spectral sequence.

TheOREM 4.2. Given $R, S, C_{*}, G$ as above, there exists a spectral sequence converging to $H^{*}\left(C_{*} ; G\right)$ with $E_{2}^{p, q} \cong \operatorname{Ext}_{R}^{q}\left(H_{p}\left(C_{*}\right), G\right)$ and differential $d_{r}$ of bidegree $(1-r, r)$. More specifically, there is a filtration:

$$
H^{m}\left(C_{*} ; G\right)=J_{m, 0} \supset J_{m-1,1} \supset \cdots \supset J_{0, m} \supset J_{-1, m+1}=0
$$

with $J_{p, q} / J_{p-1, q+1} \cong E_{\infty}^{p, q}$. All objects and isomorphisms are as right $S$-modules.

We will apply this spectral sequence with $C_{*}=C_{*}(\tilde{M}), R=S=G=\Lambda$, in order to obtain information about $H_{*} \tilde{M}$. The $E_{2}$-term is given by $E_{2}^{p, q} \cong$ $\operatorname{Ext}_{\Lambda}^{q}\left(H_{p} \tilde{M}, \Lambda\right)$. Now, $H_{0} \tilde{M} \cong \Lambda /\left(x_{1}-1, \ldots, x_{m}-1\right) \cong \mathbf{Z}$, and $H_{1} \tilde{M} \cong F_{m}^{\prime} / F_{m}^{\prime \prime}$. We analyze the spectral sequence for small $p$.

LemmA 4.3. (i) $\operatorname{Ext}_{\Lambda}^{k}(\mathbf{Z}, \Lambda) \cong 0$ unless $k=m$, and $\operatorname{Ext}_{\Lambda}^{m}(\mathbf{Z}, \Lambda) \cong \mathbf{Z}$.

(ii) $\operatorname{Ext}_{\Lambda}^{k}\left(F_{m}^{\prime} / F_{m}^{\prime \prime}, \Lambda\right) \cong 0$ unless $k=0$ or $m-2$.

If $m>2$, then $\operatorname{Ext}_{\Lambda}^{m-2}\left(F_{m}^{\prime} / F_{m}^{\prime \prime}, \Lambda\right) \cong \mathbf{Z}$ and if $m=2$, then $F_{m}^{\prime} / F_{m}^{\prime \prime} \cong \Lambda$ so that $\operatorname{Ext}_{\Lambda}^{0}\left(F_{m}^{\prime} / F_{m}^{\prime \prime}, \Lambda\right) \cong \Lambda$.

(iii) The differential $d_{2}^{1, m-2}: E_{2}^{1, m-2} \rightarrow E_{2}^{0, m}$ is an isomorphism if $m>2$ and onto if $m=2$.

Proof. The first statement follows from using the Koszul complex of $\left(x_{1}-\right.$ $\left.1, \ldots, x_{m}-1\right)$ as a free resolution of $\mathbf{Z}$. It is well known and easy to prove that the Koszul complex is self-dual up to sign. The second follows from the exact sequence:

$$
0 \rightarrow F_{m}^{\prime} / F_{m}^{\prime \prime} \rightarrow \Lambda^{m} \rightarrow \Lambda \rightarrow \mathbf{Z} \rightarrow 0
$$

The last statement is proved using the same kind of argument as that used in proving Proposition 3.3. We map $\tilde{V}$ into $\tilde{M}$ and note that the situation wanted occurs in the spectral sequence for $\tilde{V}$ for trivial dimensional reasons.

We will need to use an equivariant form of Poincaré duality, due to Milnor (see [16, Lemma 1]). Let $\tilde{Y} \rightarrow Y$ be a regular covering of a compact triangulated manifold $Y^{d}$ with boundary. If $\pi$ is the group of covering transformations, let $\lambda \mapsto \bar{\lambda}$ be the anti-automorphism of $\mathbf{Z}[\pi]$ which extends the map $g \mapsto g^{-1}$ for $g \in \pi$. We can use this to convert any right $Z[\pi]$-module $K$ to a left module and vice-versa by using the same underlying abelian group and the formula $g \cdot x=x \cdot g^{-1}$ for $x \in K, g \in \pi$. We denote the new module by $\bar{K}$.

LEMMA 4.4. There are isomorphisms of left $\mathbf{Z}[\pi]$-modules

$$
H_{d-k} \tilde{Y} \cong \overline{H_{e}^{k}(\tilde{Y}, \partial \tilde{Y} ; \mathbf{Z}[\pi])}
$$


where

$$
H_{e}^{k}(\tilde{Y}, \partial \tilde{Y} ; \mathbf{Z}[\pi])=H^{k}\left(\operatorname{Hom}_{\mathbf{Z}[\pi]}\left(C_{*}(\tilde{Y}, \partial \tilde{Y}), \mathbf{Z}[\pi]\right)\right) .
$$

We now return to the study of $H_{*} \tilde{M}$, where $\tilde{M}$ is the universal abelian cover of the closed manifold $M$ obtained from $S^{n+2}$ by surgery on the link. Recall that we have a simple link, and that $n=2 q-1$ with $q \geqslant 2$. The first result says that $H_{q} \tilde{M}$ has a particularly nice presentation as a $\Lambda$-module.

THEOREM 4.5. $H_{q} \tilde{M}$ has projective dimension one. Since $H_{q} \tilde{M}$ is of type $L$, and hence a $\Lambda$-torsion module, this is equivalent to the statement that $H_{q} \tilde{M}$ is presented by a square matrix over $\Lambda$ with determinant $\Delta$ and $\varepsilon(\Delta)= \pm 1$. by

Proof. First, assume $q>2$. Then by Lemma 4.1, a presentation of $H_{q} \tilde{M}$ is given

$$
C_{q+1} \tilde{M} \underset{d_{q+1}}{\rightarrow} C_{q} \tilde{M} \rightarrow H_{q} \tilde{M} \rightarrow 0 .
$$

To prove the theorem for $q \geq 2$, it will suffice to show that $H_{q+1} \tilde{M} \cong 0$. But by the previous lemma, $H_{q+1} \tilde{M} \cong \overline{H_{e}^{q}(\tilde{M} ; \Lambda)}$. The terms in the $E_{\infty}$-term of the universal coefficient spectral sequence which would filter $H_{e}^{q}(\tilde{M} ; \Lambda)$ are already zero in $E_{3}$, by Lemma 4.3. Hence, $H_{e}^{q}(\tilde{M} ; \Lambda) \cong 0$ and so $H_{q+1} \tilde{M} \cong 0$.

For $q=2$, we claim that, by the same argument, $H_{q+1} \tilde{M} \cong 0$. We need to prove that in $C_{*} \tilde{M}, d_{4}: C_{4} \tilde{M} \rightarrow C_{3} \tilde{M}$ and $d_{2}: C_{2} \tilde{M} \rightarrow C_{1} \tilde{M}$ are both the zero map. We will accomplish this using a series of rank-counting arguments.

First, $C_{1} \tilde{M} \cong \Lambda^{m}, C_{0} \tilde{M} \cong \Lambda, H_{1} \tilde{M} \cong F_{m}^{\prime} / F_{m}^{\prime \prime}$ and $H_{0} \tilde{M} \cong \mathbf{Z}$. If rank $d_{2}$ is greater than 0 , then $H_{1} \tilde{M}$ would have rank less than $m-1$. This would be because rank $d_{1}=1$, so that ker $d_{1}$ would have rank $m-1$. This, however, would contradict Lemma 2.5. Hence, $d_{2}$ is the zero map. By duality, $H_{4} \tilde{M}$ has rank $m-1$. Since $H_{5} \tilde{M} \cong 0$, we have:

$$
\begin{aligned}
& C_{5} \tilde{M} \quad \underset{d_{5}}{\rightarrow} C_{4} \tilde{M} \quad \underset{d_{4}}{\rightarrow} C_{3} \tilde{M} \\
& \downarrow \cong \quad \downarrow \cong \\
& \Lambda \quad \Lambda
\end{aligned}
$$

where $d_{5}$ is injective. If rank $d_{4}>0$, then rank $H_{4} \tilde{M}<m-1$ by a similar argument to the above. Hence, $d_{4}$ and $d_{2}$ are zero maps. This means that the argument for the case $q>2$ works for this case, also. We rephrase all this in terms of $\tilde{X}$.

COROllary 4.6. If $X$ is the complement of a simple boundary link of dimension $n=2 q-1$, then $H_{q} \tilde{X}$ has projective dimension one. In other words, combining this with Theorem 3.1, $H_{q} \tilde{X}$ is presented as a $\Lambda$-module by a square matrix with entries in $\Lambda$ whose determinant $\Delta$ satisfies $\varepsilon(\Delta)= \pm 1$.

We now show that the Blanchfield pairing on $H_{q} \tilde{X}$ is nonsingular. Since $\tilde{X}$ is $(2 q+1)$-dimensional, one can define the (equivariant) linking pairing of $q$-dimensional cycles (see [16])

$$
H_{q} \tilde{X} \times H_{q}(\tilde{X}, \partial \tilde{X}) \rightarrow \Lambda_{0} / \Lambda
$$


where $\Lambda_{0}$ is the quotient field of $\Lambda$. This pairing is linear in the first variable, conjugate linear in the second (using the conjugation $\lambda \rightarrow \bar{\lambda}$ defined earlier) and $\varepsilon$-Hermitian where $\varepsilon=(-1)^{q+1}$ (meaning $\langle\alpha, \beta\rangle=\overline{\varepsilon\langle\beta, \alpha\rangle}$ ). However, since $H_{q} \tilde{X}$ $\cong H_{q}(\tilde{X}, \partial \tilde{X})$, we may consider this to be a pairing on $H_{q} \tilde{X}$. One can define this pairing (or rather, its adjoint) algebraically by the following composition:

$$
\overline{H_{q} \tilde{X}} \underset{\approx}{\rightarrow} \overline{H_{q}(\tilde{X}, \partial \tilde{X})} \cong H_{e}^{q+1}(\tilde{X} ; \Lambda) \underset{\approx}{\stackrel{\delta^{*}}{\leftarrow}} H_{e}^{q}\left(\tilde{X} ; \Lambda_{0} / \Lambda\right) \underset{\psi}{\rightarrow} \operatorname{Hom}_{\Lambda}\left(H_{q} \tilde{X}, \Lambda_{0} / \Lambda\right)
$$

The first map is inclusion, the second is the duality of Lemma 4.4, the third map $\delta^{*}$ is the coboundary in the exact sequence of coefficient sequence $0 \rightarrow \Lambda \rightarrow \Lambda_{0} \rightarrow$ $\Lambda_{0} / \Lambda \rightarrow 0$, and $\psi$ is the evaluation map. All maps are easily seen to be isomorphisms except $\psi$. For this we use the spectral sequence of Theorem 4.2 with $C_{*}=C_{*} \tilde{X}, R=S=\Lambda, G=\Lambda_{0} / \Lambda$.

LEMMA 4.7. The evaluation map $\psi: H_{e}^{q}\left(\tilde{X} ; \Lambda_{0} / \Lambda\right) \rightarrow \operatorname{Hom}_{\Lambda}\left(H_{q} \tilde{X}, \Lambda_{0} / \Lambda\right)$ is an isomorphism.

Proof. The edge homomorphism $H_{e}^{q}\left(\tilde{X} ; \Lambda_{0} / \Lambda\right) \rightarrow E_{\infty}^{q, 0}$ is the evaluation map. From arguments similar to those used to prove Lemma 4.3, we see that all the other terms $E_{\infty}^{t, s}$ with $r+s=q$ are zero. Hence, $\psi$ is an isomorphism.

We have proved an analogue of Blanchfield duality.

COROllaRY 4.8. The linking pairing induces a nonsingular $\varepsilon$-Hermitian pairing on $H_{q} \tilde{X}$ taking values in $\Lambda_{0} / \Lambda$. The nonsingularity refers to the fact that the adjoint $H_{q} \tilde{X} \rightarrow \operatorname{Hom}_{\Lambda}\left(H_{q} \tilde{X}, \Lambda_{0} / \Lambda\right)$ is an isomorphism.

5. Realization below the middle dimension. In this section, we show how to realize certain modules of type $L$ as $H_{q} \tilde{X}, q \leqslant[n / 2]$. The main result of this section is:

THeOREM 5.1. Let $A_{1}, \ldots, A_{q}$ be any sequence of $\Lambda$-modules such that:

(i) $A_{i}$ is of type $L$.

(ii) For each $i, A_{i}$ has projective dimension one.

Then, for any $n \geqslant \max \{2 q, 3\}$, there exists a boundary link of $n$-spheres in $S^{n+2}$ with $H_{1} \tilde{X} \cong F_{m}^{\prime} / F_{m}^{\prime \prime} \oplus A_{1}$ and $H_{k} \tilde{X} \cong A_{k}$ for $2 \leqslant k \leqslant q$.

The proof will consist of two main parts. First, we construct a CW-complex $P$ with all the desired homological properties, and then we use $P$ to construct our link. The technique is an adaptation of a technique due to Wall and outlined by Levine in [14] for constructing knots. The proof is similar to that in [18].

LemMA 5.2. Let $k$ be an integer between 2 and $q$, inclusive. There exists a finite $C W$-complex $P$ of dimension $k+1$ such that

(i) $\pi_{1} P \cong F_{m}$.

(ii) The 1-skeleton of $P$ is $V$ (see §2).

(iii) $H_{i} \tilde{P} \cong 0$ unless $i=0,1$ or $k$, and $H_{k} \tilde{P} \cong A_{k}$, where $\tilde{P}$ is the universal abelian cover of $P$.

(iv) $H_{*} P \cong H_{*} V$. 
Proof. We will build $P$ by realizing a presentation of $A_{k}$ in the chains of $\tilde{P}$. Thus, let us choose a presentation $0 \rightarrow F_{1} \rightarrow{ }_{d} F_{0} \rightarrow A_{k} \rightarrow 0$. The $F_{i}$ are free modules over $\Lambda$ of the same rank $r$. Let $\left\{e_{1}, \ldots, e_{r}\right\}$ be a basis for $F_{1}$.

Begin with $V$ as the 1 -skeleton. To $V$, wedge on a bouquet of $r k$-spheres, forming $W$. We have $C_{k} \tilde{W} \cong F_{0}$. Since $\pi_{1} W$ is free and $\pi_{i} W \cong 0$ for $1<i<k$, it is not difficult to see that the Hurewicz map $\pi_{k} \tilde{W} \rightarrow H_{k} \tilde{W} \cong C_{k} \tilde{W}$ is onto. Choose maps $f_{1}, \ldots, f_{r}: S^{k} \rightarrow W$ corresponding to $d\left(e_{1}\right), \ldots, d\left(e_{r}\right)$ under the composition:

$$
\pi_{k} W \longleftarrow \pi_{k} \tilde{W} \rightarrow H_{k} \tilde{W} \cong F_{0}
$$

Attach $(q+1)$-cells to $W$ via these maps to form $P$. It is easy to see that (i)-(iii) are satisfied. To see (iv), we merely note that, since $A_{k}$ is of type $L$, the map $1 \otimes_{\Lambda} d: \mathbf{Z} \otimes_{\Lambda} C_{k+1} \rightarrow \mathbf{Z} \otimes_{\Lambda} C_{k}$ is an isomorphism. This completes the proof.

Lemma 5.3. There exists a finite $C W$-complex $P$ of dimension $q+1$ such that:

(i) $\pi_{1} P \cong F_{m}$.

(ii) The 1-skeleton of $P$ is $V$.

(iii) $H_{k} \tilde{P} \cong A_{k}$ for $2 \leqslant k \leqslant q$ and $H_{k} \tilde{P} \cong 0$ for $k>q$.

(iv) $H_{*} P \cong H_{*} V$.

Proof. For each $k$, let $W_{k}$ be the complex constructed in Lemma 5.2 of dimension $k+1$. $P$ is formed from the disjoint union of the $W_{k}$ by identifying their 1-skeletons. One may easily check that $P$ has all the desired properties.

The next step is to construct a group $\pi$ with the necessary properties. First, we prove a lemma which will justify one of the conditions we wish to place on $\pi$.

LEMMA 5.4. Let $G$ be a group such that $G / G^{\prime}$ is free abelian of rank $r$ and $G$ has a presentation with $r$ more generators than relations. Then there exists a finite 2-complex $T$ with $\pi_{1} T \cong G$ and $H_{k} T \cong 0$ for $k>1$. Furthermore, if $\tilde{T}$ is the universal abelian cover of $T$, then $H_{k} \tilde{T} \cong 0$ for $k>1$.

Proof. This is all done by counting ranks. Begin with a wedge of circles to represent the generators of $G$, and attach 2-cells to represent the relations. The chain complex of $T$ is as follows:

$$
\begin{array}{ccccc}
C_{2} T & \overrightarrow{d_{2}} & C_{1} T & \underset{d_{1}=0}{\rightarrow} & C_{0} T \\
\downarrow & \underset{\mathbf{Z}^{s}}{\downarrow} \cong & & & \downarrow \\
& & \mathbf{Z}^{s+r} & & \mathbf{Z}
\end{array}
$$

Since $H_{1} T \cong \mathbf{Z}^{r}$ and ker $d_{1}=C_{1} T, d_{2}$ must be injective. This shows that $H_{k} T \cong 0$ for $k>1$. In $\tilde{T}$, the chains are given by the following, where $\Lambda \cong \mathbf{Z}\left[\mathbf{Z}^{r}\right]$ :

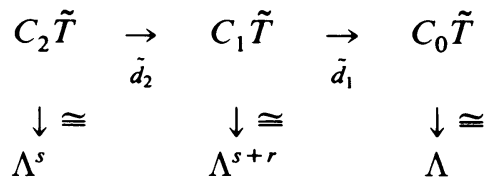

We need to show that $\tilde{d}_{2}$ is injective, or equivalently, that $\tilde{d}_{2}$ has rank $s$. But $d_{2}$ has rank $s$, and $d_{2}=1 \otimes_{\Lambda} \tilde{d}_{2}: \mathbf{Z} \otimes_{\Lambda} C_{2} \tilde{T} \rightarrow \mathbf{Z} \otimes_{\Lambda} C_{1} \tilde{T}$, so the rank of $\tilde{d}_{2}$ must be at least as great as the rank of $\tilde{d}_{2}$. Hence, $\tilde{d}_{2}$ is injective. This shows that $H_{k} \tilde{T} \cong 0$ for $k>1$. 
We will want to pass back and forth between the free group on $m$ generators $F_{m}$ and the free abelian group $Z^{m}$, so we will choose specific inverse images in $F_{m}$ of elements of $\mathbf{Z}^{m}$. Let $x_{1}, \ldots, x_{m}$ be the generators of $F_{m}$; elements of the form $x_{1}^{l_{1}} \cdot x_{2}^{l_{2}} \ldots x_{m}^{l_{m}}$ will be called the lexic elements of $F_{m}$. They may be specified by an $m$-tuple of integers, and there is exactly one lexic element of $F_{m}$ for any element of $\mathbf{Z}^{m}$.

It is clear from the discussion in $\S 1$ that if $A$ is any $\Lambda$-module of type $L$ with projective dimension one, then $A$ is presented by a square matrix over $\Lambda$ whose determinant augments to 1 (that is, there exists a matrix $\left(\lambda_{i j}\right)$ over $\Lambda$ such that the integral matrix $\left(\varepsilon\left(\lambda_{i j}\right)\right)$ has determinant 1$)$. In fact, we can prove the following:

LEMMA 5.5. $\left(\lambda_{i j}\right)$ may be chosen so that $\varepsilon\left(\lambda_{i j}\right)=\delta_{i j}$.

Proof. This is because any integral matrix with determinant 1 can be transformed to the identity via row and column operations. Doing the same operations to the original matrix $\left(\lambda_{i j}\right)$ does not change the isomorphism class of the module it presents, and clearly gives us the matrix we desire.

We are now in a position to state the main result concerning the group $\pi$.

TheOREM 5.6. Let $A_{1}$ be any module of type $L$ with projective dimension one. Then there exists a group $\pi$ satisfying:

(i) $\pi$ has a finite presentation with $m$ more generators than relations.

(ii) $\pi$ has weight $m$, i.e. there exist $m$ elements $\mu_{1}, \ldots, \mu_{m} \in \pi$ such that the normal subgroup generated by the $\mu_{i}$ is all of $\pi$, and there exists no set having this property with fewer elements.

(iii) $\pi / \pi^{\prime} \cong \mathbf{Z}^{m}$.

(iv) $\pi^{\prime} / \pi^{\prime \prime} \cong F_{m}^{\prime} / F_{m}^{\prime \prime} \oplus A_{1}$ as a $\Lambda$-module.

(v) There exists a surjection of $\pi$ onto $F_{m}$ which sends the elements $\mu_{i}$ of (ii) onto the free generators of $F_{m}$.

REMARKS. The group $\pi$ will be the fundamental group of the complement of our link. An application of Lemma 5.4 tells us that $\pi$ has no undesirable homological properties. By a result of Kervaire [10] conditions (i), (ii) and (iii) imply that $\pi$ is the group of a link of $n$-spheres in $S^{n+2}$ for any $n \geqslant 3$. Condition (v) will insure that the link we construct will be a boundary link, by Lemma 2.1, as the $\mu_{i}$ will be the elements represented by the meridians.

Proof of Theorem 5.6. Choose a presentation matrix $\left(a_{i j}\right)$ for $A_{1}$ of the type given by Lemma 5.5. A presentation for $\pi$ is given by:

$$
\pi=\left(x_{1}, x_{2}, \ldots, x_{m}, t_{1}, t_{2}, \ldots, t_{r} \mid \prod_{j, g}\left(g t_{j} g^{-1}\right)^{\lambda_{i j g}}=1 \text { for } i=1, \ldots, r\right)
$$

where $\left(a_{i j}\right)$ is an $r \times r$ matrix, $a_{i j}=\Sigma_{g} \lambda_{i j g} g \in \Lambda$, and $g$ varies over the lexic elements of the free group $F_{m}$ generated by the $x_{i}$. The elements in the product are in lexical order determined by the $(m+1)$-tuple $\left(j, l_{1 g}, l_{2 g}, \ldots, l_{m g}\right)$ where $g=$ $x_{1}^{l_{18}} x_{2}^{l_{28}} \ldots x_{m}^{l_{m g}}$. Condition (i) is clearly satisfied. We obtain the following abelian 
presentation for $\pi / \pi^{\prime}$, where we write the relations additively:

$$
\pi / \pi^{\prime}=\left(x_{1}, \ldots, x_{m}, t_{1}, \ldots, t_{r} \mid \sum_{j, g} \lambda_{i j g} t_{j}=0, \quad i=1, \ldots, r\right) .
$$

But $\sum_{j, g} \lambda_{i j g} t_{j}=\sum_{j}\left(\Sigma_{g} \lambda_{i j g}\right) t_{j}=\Sigma_{j} \varepsilon\left(a_{i j}\right) t_{j}=\Sigma_{j} \delta_{i j} t_{j}=t_{i}$ so we can simplify the presentation for $\pi / \pi^{\prime}: \pi / \pi^{\prime}=\left(x_{1}, \ldots, x_{m}, t_{1}, \ldots, t_{r} \mid t_{i}=0, i=1, \ldots, r\right)$. Hence, $\pi / \pi^{\prime} \cong \mathbf{Z}^{m}$, proving (iii). It is easy to see that the weight of $\pi$ must therefore be at least $m$. If we adjoin the relations $x_{1}=x_{2}=\cdots=x_{m}=1$ to $\pi$, the group becomes $\left(t_{1}, \ldots, t_{r} \mid \Pi_{j, g} t_{j}^{\lambda_{j g}}=1, i=1, \ldots, r\right)$. But $\Pi_{j, g} t_{j}^{\lambda_{j / g}}=\Pi_{j} t_{j}^{\Sigma \lambda_{j, g}}$ by virtue of the fact that elements in the product are in lexical order. Since $\sum \lambda_{i j g}=\varepsilon\left(a_{i j}\right)=\delta_{i j}$, the group is given by $\left(t_{1}, \ldots, t_{r} \mid t_{i}=1, i=1, \ldots, r\right)=(e)$. Hence, $\pi$ has weight $m$. To see (v), adjoin the relations $t_{1}=t_{2}=\cdots=t_{r}=1$ to $\pi$. This gives the free group generated by the $x_{i}$.

Since $\pi / \pi^{\prime}$ is the free abelian group on the $x_{i}$, it follows that $\pi^{\prime}$ is the normal subgroup of $\pi$ generated by the commutator subgroup of $F_{m}$ and the $t_{j}$. In fact, a presentation of $\pi^{\prime}$ is given by

$$
\pi^{\prime}=\left(\left\{t_{i, g}\right\}, F_{m}^{\prime} \mid \prod_{j, g}\left(t_{i, g}\right)^{\lambda_{i j g}}=1, h t_{i, g} h^{-1}=t_{i, h g}\right)
$$

where we set $t_{i, g}=g t_{i} g^{-1}, g$ and $h$ vary over all of $F_{m}$ and we understand that $\lambda_{i j g}=0$ if $g$ is not lexic. Using the equation $g \cdot T_{j}=t_{i, g}$ via the action of $F_{m}$ on $\pi^{\prime} / \pi^{\prime \prime}$ to define the $T_{j}$, we obtain the following presentation for $\pi^{\prime} / \pi^{\prime \prime}$ as a $\Lambda$-module:

$$
\pi^{\prime} / \pi^{\prime \prime}=\left(\left\{T_{j}\right\}, F_{m}^{\prime} \mid \sum_{j, g} \lambda_{i j g} g \cdot T_{j}=0, F_{m}^{\prime \prime}\right) .
$$

Since $\sum_{j, g} a_{i j g} g \cdot T_{j}=\Sigma_{j}\left(\sum_{g} a_{i j g} g\right) \cdot T_{j}=\Sigma_{j} a_{i j} T_{j}$, we get the isomorphism of $\Lambda$ modules $\pi^{\prime} / \pi^{\prime \prime} \cong F_{m}^{\prime} / F_{m}^{\prime \prime} \oplus A_{1}$ which completes the proof.

Now let $T$ be a 2-complex realizing $\pi$ as in Lemma 5.4. Let $f: V \rightarrow T$ be the map which sends the loops of $V$ to loops in $T$ representing the $x_{i} \in \pi=\pi_{1} T$, and form the complex $K$ from the disjoint union of $P$ (from Lemma 5.3) and $T$ via the map $f$. It is easy to verify the following lemma.

LEMMA 5.7. $K$ is a finite $(q+1)$-dimensional $C W$-complex satisfying:

(i) $H_{*} K \cong H_{*} V$.

(ii) $\pi_{1} K \cong \pi$.

(iii) $H_{1} \tilde{K} \cong F_{m}^{\prime} / F_{m}^{\prime \prime} \oplus A_{1}, H_{k} \tilde{K} \cong A_{k}$ for $2 \leqslant k \leqslant q$ and $H_{k} \tilde{K} \cong 0$ for $k>q$.

We now go to the second part of the proof of Theorem 5.1. We use a result of Levine [14, Lemma 10.1].

LeMmA 5.8. $K$ embeds in $\mathbf{R}^{n+3}$, where $n$ is any integer greater than or equal to $\max \{2 q, 3\}$. Let $M$ be the boundary of a regular neighborhood $N(K)$. Then $M$ has nonzero homology only in dimensions $0,1,(n+1)$ and $(n+2)$. The following composite map is $(n+1-q)$-connected:

$$
M \underset{\text { inclusion }}{\rightarrow} N(K) \underset{\text { retraction }}{\rightarrow} P .
$$


We now prove Theorem 5.1. Choose embeddings $\varphi_{1}, \ldots, \varphi_{m}: S^{\prime}: D^{n+1} \rightarrow M$ with disjoint images representing the elements $x_{1}, \ldots, x_{m} \in \pi \cong \pi_{1} M$. Surgery on these maps yields a manifold $\Sigma^{n+2}$ and embeddings $\psi_{1}, \ldots, \psi_{m}: D^{2} \times S^{n} \rightarrow \Sigma^{n+2}$ with disjoint images. The manifold $\Sigma$ is easily seen to be a simply-connected homology sphere, hence a homotopy sphere. Since $n+2 \geqslant 5$, we can change the differentiable structure of $\Sigma$ in the neighborhood of a point to obtain $S^{n+2}$, by arguments of Smale [20]. The link we want is given by the embeddings $\psi_{i}\left(0 \times S^{n}\right)$. The complement of the link is equal to the space $X=\overline{M-\cup_{i} \text { im } \varphi_{i}}$ and the pair $(M, X)$ is $n$-connected by general position. Hence, $X$ has all the homological properties as specified.

6. Polynomial invariants. We now relate the material of the preceding sections to the polynomial invariants of a link. Our definition will differ slightly from Levine's definition (see [12]) for knots. First, in a more general setting, the elementary divisors will be defined.

As in $\S 1$, if $M$ is an $R$-module, one has the Fitting ideals $I_{i}(M)$. If $R$ is a unique factorization domain, then any ideal of $R$ is contained in a unique minimal principal ideal (generated by the greatest common divisor). Let $\Delta_{i}(M)$ be a generator of the smallest principal ideal containing $I_{i}(M)$. Then $\Delta_{i+1} \mid \Delta_{i}$ for each $i$, and $\Delta_{i}(M)=1$ for $i$ sufficiently large.

Now suppose we have a link $L$ of $n$-spheres in $S^{n+2}$. Since $\Lambda$ is a unique factorization domain and $H_{k} \tilde{X}$ is finitely generated for each $k$, we may define the polynomial invariants of $L$ to be the elements defined by $p_{j, k}=\Delta_{j}\left(H_{k} \tilde{X}\right)$ where $0 \leqslant j$ and $1 \leqslant k \leqslant n$. We justify the terminology as follows. The $\Delta_{i}$ are defined only up to multiplication by units of $\Lambda$. Every element of $\Lambda$ can be written as the product of a polynomial and a unit of $\Lambda$. Therefore, up to the indeterminacy in the definition of the $\Delta_{i}$, all elements of $\Lambda$ are polynomials.

We now interpret some of the results we have proved in the language of polynomial invariants.

THEOREM 6.1. Suppose that $L$ is a boundary link of $n$-spheres in $S^{n+2}$, and $p_{j, k}(L)$ are its polynomial invariants. Then

(i) $p_{j, 1}=0$ for $j<m-1$, where $m$ is the number of components of $L$.

(ii) $p_{j, k}(1,1, \ldots, 1)= \pm 1$ for all $j$ if $k>1$, and for all $j>m-1$ if $k=1$.

(iii) $p_{j+1, k} \mid p_{j, k}$ for all $j, k$.

(iv) $p_{j, k}=1$ for $j$ sufficiently large.

Conversely, suppose $\left\{p_{j, k}\right\}, j \geqslant 0$ and $1 \leqslant k \leqslant q$, is a collection of polynomials satisfying (i)-(iv) above. Then for any $n \geqslant \max \{2 q, 3\}$, there exists a boundary link $L$ of $n$-spheres in $S^{n+2}$ with $p_{j, k}(L)=p_{j, k}$ for $j \geqslant 0,1<k \leqslant q$.

If $L$ is a simple boundary link of dimension $n=2 q-1, q \geqslant 2$, then

(v) $p_{q}\left(1 / x_{1}, 1 / x_{2}, \ldots, 1 / x_{m}\right)=u \cdot p\left(x_{1}, \ldots, x_{m}\right)$ where $u= \pm x_{l}^{l_{1}} x_{2}^{l_{2}} \ldots x_{m}^{l_{m}}$ for some integers $l_{1}, \ldots, l_{m}$.

Proof. (i) is a consequence of Corollary 2.4, Lemma 2.5 and [1, Lemma 4.10]. (ii) follows from Lemma 2.6 and Theorem 3.1. Properties (iii) and (iv) are just consequences of the definition of elementary divisors. 
The converse is proved by constructing modules of type $L$ of projective dimension one having the desired elementary divisors and using Theorem 5.1 to construct the link.

We make use of Theorem 1.6. For each $j, k$ let $\lambda^{j, k}=p_{j, k} / p_{j+1, k}$. For $j>N$, all the $\lambda^{j, k}=1$. For each $k=1, \ldots, q$ let $A_{k}$ be the module presented by the diagonal matrix $\operatorname{diag}\left(\lambda^{0, k}, \lambda^{1, k}, \ldots, \lambda^{N, k}\right)$. Since the determinant of this matrix is $p_{0, k}, A_{k}$ is of type $L$.

In the case of a simple boundary link, we use Corollary 4.8 to deduce that $\overrightarrow{H_{q} \tilde{X}} \cong \operatorname{Hom}_{\Lambda}\left(H_{q} \tilde{X}, \Lambda_{0} / \Lambda\right)$. Application of results of Blanchfield [1, Theorems 4.4 and 4.5 ] yields $(\mathrm{v})$.

\section{REFERENCES}

1. R. Blanchfield, Intersection theory of manifolds with operators with applications to knot theory, Ann. of Math. (2) 65 (1957), 340-356.

2. D. Buchsbaum and D. Eisenbud, What makes a complex exact?, J. Algebra 25 (1973), 259-268.

3. __ Some structure theorems for finite free resolutions, Advances in Math. 12 (1974), 84-139.

4. H. Cartan and S. Eilenburg, Homological algebra, Princeton Univ. Press, Princeton, N. J., 1956.

5. W. Dwyer, Vanishing homology over nilpotent groups, Proc. Amer. Math. Soc. 49 (1975), 8-12.

6. M. Gutierrez, Boundary links and an unlinking theorem, Trans. Amer. Math. Soc. 171 (1972), 491-499.

7. __ Polynomial invariants of boundary links, Rev. Columbian Mat. 8 (1974), 97-109.

8. P. Hilton and S. Wylie, Homology theory: An introduction to algebraic topology, Cambridge Univ. Press, Cambridge, 1960.

9. I. Kaplansky, Commutative algebra, Allyn and Bacon, Boston, Mass., 1970.

10. M. Kervaire, Les noeds de dimensions supérieures, Bull. Soc. Math. France 93 (1965), 225-271.

11. J. Levine, A characterization of knot polynomials, Topology 4 (1965), 135-141.

12. ___ Polynomial invariants of knots of codimension two, Ann. of Math. (2) 84 (1966), 537-554.

13. __ A method for generating link polynomials, Amer. J. Math. 89 (1967), 69-84.

14. Knot modules. I, Trans. Amer. Math. Soc. 229 (1977), 1-50.

15. H. Matsumura, Commutative algebra, Benjamin, New York, 1970.

16. J. Milnor, A duality theorem for Reidemeister torsion, Ann. of Math. (2) 76 (1962), 137-147.

17. D. G. Northcott, Finite free resolutions, Cambridge Univ. Press, Cambridge, Mass., 1976.

18. N. A. Sato, Free coverings and modules of boundary links, Trans. Amer. Math. Soc. (to appear).

19. H. Seifert, Über das Geschlecht von Knoten, Math. Ann. 110 (1934), 571-592.

20. S. Smale, On the structure of manifolds, Amer. J. Math. 84 (1962), 387-399.

21. N. Smythe, Boundary links, Topology Seminar (Wisconsin, 1965), Princeton Univ. Press, Princeton, N. J., 1966.

22. R. G. Swan, Projective modules over Laurent polynomial rings, Trans. Amer. Math. Soc. 237 (1978), 111-120.

23. J. Smith, Acyclic localizations, J. Pure Appl. Algebra 12 (1978), 117-127.

Department of Mathematics, University of Texas, Austin, Texas 78712 